Katja Bartlakowski

\title{
Make the library really look more like google
}

\section{Zur Einführung des Discovery-Systems „scinos“ an der Hochschule Osnabrück}

Zusammenfassung: Immer noch ist es Wunsch und Überzeugung vieler Bibliotheken, dass ihr Internetauftritt als zentrale Anlaufstelle für Informationssuchende wahrgenommen wird. In der Realität hingegen spielt der Wert der Bibliothekswebsite für Studierende und Lehrende eine zunehmend untergeordnete Rolle. Die Einführung eines Discovery-Systems bietet in diesem Zusammenhang gute Möglichkeiten, das überholte Portalkonzept bibliothekarischer Internetseiten zu überdenken und neue Wege zu gehen.

Schlüsselwörter: Discovery-System, Bibliothekswebsite, Portalkonzept

\section{Introducing the "scinos" discovery system at Osnabrück University of Applied Sciences}

Abstract: Many libraries are convinced that their internet presence should continue to be a central portal for information seekers and would like it to stay this way. However, in reality the situation is completely different. Library websites play an ever-decreasing role for students and teaching staff. The introduction of a discovery system provides a good opportunity to rethink the outdated portal concept used for library internet pages websites and to break new ground. explore new avenues.

Keywords: discovery system, library website, portal concept

DOI 10.1515/bd-2015-0073

1 Der Titel dieses Beitrags ist inspiriert durch den Aufsatz von Oliver Kohl-Frey: Make the Library look more like Google - Die Einführung eines Discovery-Systems an der Universität Konstanz, http://kops.uni-konstanz.de/handle/123456789/19372 [Zugriff: 06.01.2015].

Dr. Katja Bartlakowski: K.Bartlakowski@hs-osnabrueck.de 


\section{Einleitung}

Die Website gilt aus bibliothekarischer Sicht als Startpunkt und Eingangstor zu den wissenschaftlichen Informationen, werden doch auf ihr hochkarätige Datenbanken, E-Journals und E-Books bereit gestellt. Mag das Rechercheangebot auch noch so exklusiv sein, schnell wird einem im Austausch mit Studierenden und Lehrenden klar, dass die Bibliothekswebsite häufig nicht das wichtigste Zugangsportal zu wissenschaftlicher Information ist. Viele Lehrkräfte neigen dazu, ihre bevorzugten Datenbanken und Quellen in der Favoritenliste ihres Browsers abzuspeichern; andere suchen sich eigene Wege, etwa über Google Scholar, um zum gewünschten Inhalt zu gelangen. Nicht selten wird die Bibliothekswebsite von Lehrenden und Studierenden als inhaltlich überfrachtet und wenig eingängig beschrieben. Wortabkürzungen wie etwa EZB, DBIS, OPAC sowie die Vielzahl der verschiedenen Rechercheangebote und Sucheinstiegspunkte in Gestalt verschiedener Fachdatenbanken mit kaum verständlichen Bezeichnungen verstärken das Bild. In vielerlei Hinsicht reagieren Bibliotheken hierauf mit einem Zuwachs an Informationskompetenzveranstaltungen, im Rahmen derer Studierende wie Lehrende im Umgang mit der stetig wachsenden Zahl heterogener Informationsressourcen geschult werden.

Andere wiederum setzen auf die Einführung von Resource-Discovery-Systemen, verbunden mit dem Ziel, möglichst viele Datenbank- und Volltextangebote sowie den Online-Katalog unter einer Rechercheoberfläche zu vereinen. Indem die einfache Suchzeile der Discovery-Suchmaschine googlelike in den Mittelpunkt der Website rückt, erhoffen sich Bibliothekare, die Bibliothekswebsite als erste Rechercheanlaufstelle für Studierende und Lehrende aufwerten zu können. Ob damit tatsächlich eine höhere Akzeptanz des Internetauftritts erzielt wird, bleibt allerdings zweifelhaft.

Sich von den gewohnten Strukturen zu lösen, fällt nicht jeder wissenschaftlichen Bibliothek leicht. Aber wollen Bibliotheken ihrem Dienstleistungsanspruch gerecht werden, lohnt es, den Portalgedanken bibliothekarischer Web-Präsenzen $\mathrm{zu}$ hinterfragen und neue Wege auszuprobieren. Bibliotheken tun gut daran, sich noch mehr zu Dienstleistern zu entwickeln, die ihre Angebote dort platzieren, wo sie wahrgenommen, gebraucht und dann möglicherweise auch genutzt werden. Discovery-Systeme bieten in diesem Zusammenhang vielfältige Möglichkeiten. 


\section{2 scinos - scientific information osnabrück}

scinos ist ein EBSCO-Discovery-Tool. Die Suchmaschine bringt ein leicht daher kommendes, sehr ansprechendes Design mit den EDS-basierten Funktionalitäten in Einklang. Das einladende Design verspricht ein wahres Discovery-Erlebnis. scinos funktioniert wie Google. Im Zentrum der Startseite steht eine einfache Suchzeile, die obere Menüleiste verbindet scinos mit der Bibliothek und anderen Portalen, komfortabel eingebettet findet sich dort auch der Zugang zu den Bibliothekskonten von Hochschule und Universität Osnabrück.

\section{$0 \bigcirc$ ascientific information... beta}

Die Suchmaschine der Hochschule Osnabrück

Abb. 1: Das scinos-Logo.

Die Suchmaschine vereint die Kataloge der beiden Hochschulen in Osnabrück, zudem kommerzielle Ressourcen mit internationalen Open-Access-Inhalten. Vieles ist im Volltext vorhanden. Die Anreicherung mit Grafiken, Lernvideos und anderen E-Learning-Produkten macht scinos in einem besonderen Maße für Lehrende und Studierende interessant. Der Zugang zu Informationen zur Forschungsförderung komplettiert das Bild. Obgleich noch in der Entwicklungsphase, ist scinos bereits jetzt ein attraktiver Begleiter für Studium, Forschung und Lehre.

Nicht oder nur unzureichend in scinos erschlossene Fachdatenbanken der Hochschule Osnabrück werden je nach Suchanfrage per Empfehlung auf der Trefferseite angezeigt und ermöglichen so einen schnellen und unkomplizierten Zugang zur Quelle. Dazu werden viele Suchanfragen begleitet von enzyklopädischen, zitierfähigen Beiträgen (etwa aus der Encyclopedia Britannica), die Informationen bzw. Beschreibungen zum Suchbegriff liefern und damit einen thematischen Einstieg ermöglichen. Dieser so genannte „Research Starter“ ist eine interessante und wissenschaftlich unbedenkliche Alternative zur Wikipedia. Entsprechende Vorlese- und Übersetzungsfunktionen machen die Texte zudem barrierefrei zugänglich.

Als zusätzlichen Service sollen perspektivisch wesentliche Inhalte aus den Web-Präsenzen von Bibliothek und Hochschule sowie weitere Informationen aus der studentischen Lebenswelt in scinos erfasst und damit auffindbar werden. Die 
Suche nach den Öffnungszeiten der Bibliothek wird dann ebenso zum Ergebnis führen wie etwa die Suche nach dem Speiseplan der Mensa.

Der EDS enthält vielfältige Tools. Alle bibliographischen Daten lassen sich im Einzelaufruf bequem weiter verarbeiten, etwa abspeichern, per Mail weiterleiten, ausdrucken oder in ein Literaturverwaltungsprogramm wie etwa Citavi überführen. Auch können die Informationen in sozialen Netzwerken wie Facebook, Twitter, XING etc. geteilt werden.

Die integrierte Funktion der mobilen Suche rundet das Profil ab. scinos erkennt das mobile Endgerät und passt seine Funktionalitäten automatisch an.

\section{Abkehr vom Portalgedanken der Bibliothekswebsite}

scinos wird an der Hochschule Osnabrück in mehreren Phasen eingeführt. Die Entwicklung des Produktes ist bei Weitem noch nicht abgeschlossen. Gleichwohl ist scinos bereits in einer Beta-Version online und wird derzeit als zunächst zusätzlicher Dienst auf der Website der Bibliothek angeboten. scinos bleibt jedoch kein „Zweitkatalog“.

In der zweiten Einführungsphase wird scinos die bislang bestehenden heterogenen Informationszugänge vordergründig ablösen: scinos wird das neue Frontface der Bibliothek. Die Bibliothekswebsite rückt in den Hintergrund und verliert so ihren Charakter eines zentralen Einstiegssportals zu wissenschaftlichen Informationen. Das ursprüngliche Portalkonzept wird aufgegeben. Im Gegensatz zur Website ist scinos schnell erfassbar, weitestgehend intuitiv bedienbar und auf das Wesentliche reduziert. Eben unkompliziert. 

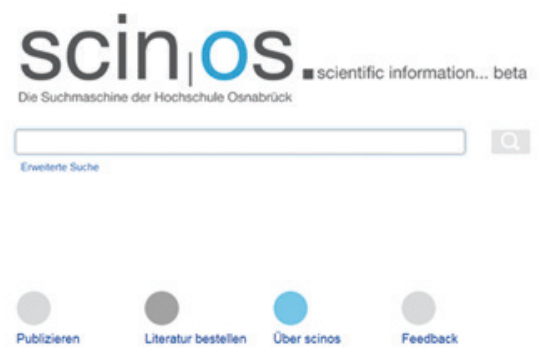

Abb. 2: Die scinos-Startseite.

Ziel ist es, in einem dritten Schritt scinos überall dort zu verankern, wo Studierende und Lehrende das Angebot leicht finden können. Hierfür bieten sich Fakultätswebsites und das Campusmanagementsystem der Hochschule genauso an wie etwa die an der Hochschule etablierten E-Learning- oder Kommunikationsplattformen. Parallel hierzu ist es geplant, die Website der Bibliothek grundsätzlich zu überarbeiten und in der Folge vermehrt Dienste der Bibliothek dort anzubieten, wo sie auch wahrgenommen werden. Mit der flächendeckenden Vernetzung der „Bibliothek“ in der Hochschulwelt erhoffen wir uns eine bessere, hochschulweite Präsenz der bibliothekarischen Dienstleistungen.

Die Angebote wissenschaftlicher Bibliotheken sind unbestritten gut. Gleichwohl genügt es nicht mehr, darauf zu warten, dass Studierende oder Lehrende die Vielfalt der Bibliotheksangebote entdecken. Es wird immer wichtiger, dafür zu sorgen, dass die Informationen und Services zum Kunden gelangen. Verschiedene Wege sind hier denkbar. Und ob der Weg, den die Bibliothek der Hochschule Osnabrück beschreitet, sich als gut und richtig erweisen wird, wird sich erst nach der Realisierung des Projektes und seiner Evaluation herausstellen. Bis dahin bleibt es ein spannender Prozess.

\section{Gedanken zum Abschluss}

Bereits seit vielen Jahren sind Discovery-Systeme in wissenschaftlichen Bibliotheken im Einsatz. Die Notwendigkeit einer Vereinheitlichung der heterogenen 
Informationsstruktur ist seit Langem erkannt. Dennoch beobachtet man in der Bibliothekscommunity immer wieder, dass sich das entwickelte Discovery-System als „Zweitkatalog“ auf den Webseiten wiederfindet und als zusätzliches Recherchetool angeboten wird. Liegt der Grund darin, dass ein Discovery-System niemals den Grad der bibliothekarisch gewünschten Perfektion erreichen wird und man es deshalb nur als Zusatzangebot, als „KatalogPlus“, präsentieren mag? Wenn auch der Informationsspezialist die Unschärfen und Tücken einer Discovery-Recherche rasch erkennt, der Durchschnittsnutzer wird sich über die leichte Bedienbarkeit der Suchmaschine freuen und mit der Qualität der Suchergebnisse zufrieden sein. Kommt es darauf nicht an?

Oder liegen die Ursachen für den Einsatz des Discovery-Systems als „Zweitkatalog“ etwa ganz woanders? Im Dialog mit Fachkollegen über das Thema schwingt nicht selten eine unterschwellige Ambivalenz mit. Auf der einen Seite herrscht eine große Einigkeit darüber, die Zugänge zu wissenschaftlicher Information bedienungsfreundlicher zu gestalten. Auf der anderen Seite gewährt die Komplexität der Zugänge durchaus Vorteile für den Berufsstand. Denn ihre Handhabung erfordert ein Expertenwissen und rechtfertigt augenscheinlich einmal mehr die Notwendigkeit der Informationskompetenzvermittlung in Form von (Massen-)Veranstaltungen, die seit Jahren Hochkonjunktur haben. Vielen Bibliothekaren ist es bewusst oder sie ahnen es zumindest, dass diese Dienstleistung nicht selten am Kundeninteresse vorbeizielt und mehr dem beruflichen Selbsterhalt zu dienen scheint. Die googlegeprägte Studentengeneration erwartet es nicht, geschult oder trainiert zu werden, um an die gewünschte Information zu kommen - „digital natives“ erwarten den Volltext auf Knopfdruck. Diese Realität ist gegenwärtig, mag man sie auch für schlecht befinden.

Aber vielleicht lädt uns gerade diese Realität ein, künftig weniger darüber nachzudenken, wie wir unsere Studierenden trainieren sollen, als vielmehr darüber, wie wir uns schulen können, nämlich im Ernst- und Wahrnehmen der Bedürfnisse und Lebensrealitäten unserer Kunden.

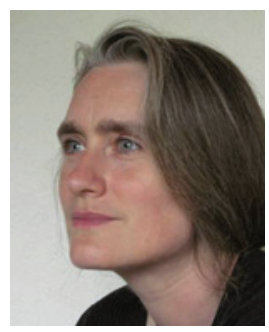

\section{Dr. Katja Bartlakowski}

Leitung Bibliothekssystem

Hochschule Osnabrück

Albrechtstr. 30

49076 Osnabrück

E-Mail: K.Bartlakowski@hs-osnabrueck.de 